\title{
Expanding the crystal form landscape: emerging computational tools for the discovery of eutectic composites and higher-order cocrystals of organic compounds
}

\section{Zeinab M. Saeed ${ }^{1}$, Bhausaheb Dhokale ${ }^{1}$, Abeer F. Shunnar², Durga Prasad Karothu², Panče Naumov², Hector H. Hernandez ${ }^{1}$ and Sharmarke Mohamed ${ }^{1}$}

${ }^{I}$ Department of Chemistry, Green Chemistry \& Materials Modelling Laboratory, Khalifa University of Science and Technology, Abu Dhabi, UAE, ${ }^{2}$ Smart Materials Laboratory, New York University Abu Dhabi, Abu Dhabi, UAE.

\author{
sharmarke.mohamed@ku.ac.ae
}

Computational methods for predicting the crystal structures [1] of organic compounds have evolved over the past three decades to the point where they are now used in major pharmaceutical companies to support the solid-form development of new active pharmaceutical ingredients (APIs). More broadly, knowledge of the crystal structure of a compound is fundamental to understanding the mechanical response, charge carrying capacity and porosity of the material. Most crystal structure prediction (CSP) studies produce a static crystal form landscape (CFL) which depicts the possible polymorphs as a function of lattice energy and crystal density. However, the challenge of extracting the set of molecular and crystal descriptors from the CFL that will support the targeted crystallization of a desired polymorph remains a major unresolved challenge within the CSP community.

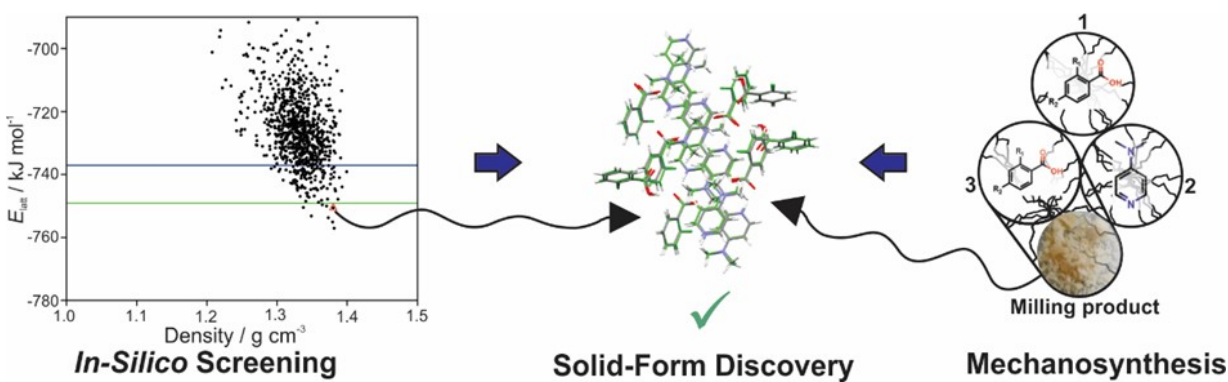

Figure 1. We apply a synergistic mechanochemical milling and in-silico screen to discover novel eutectics and higher-order crystals

Whilst there have been many reports of the application of computational methods to support the discovery of binary cocrystals, very little is known about the accuracy of CSP methods for supporting the discovery of periodic multicomponent crystals that contain $>2$ distinct chemical fragments in the crystallographic asymmetric unit. The discovery of such higher-order cocrystals (HOCs) widens the CFL and allows drug developers to choose the optimal solid dosage form for a particular API. We demonstrate [2] that CSP methods can be adapted to support the discovery of ternary molecular ionic cocrystals comprising many competing intermolecular hydrogen bonding interactions in the crystal. Beyond periodic HOCs, a eutectic composite is an example of a solid mixture that can be described as a conglomerate of solid solutions. The discovery of eutectics is associated with a depression in the melting point of the API. The extent of melting point depression is correlated with a commensurate increase in the solubility of the API. Since a large fraction of pharmaceutical lead compounds are abandoned due to poor solubility profiles, a computational model that can accurately predict eutectic formation is of significant value to the pharmaceutical industry. We demonstrate [3] that the computed mixing energies and binding modes of candidate molecular pairs leads to temperature-dependent interaction parameters that can accurately predict the formation of eutectic composite materials of molecular compounds. Our results demonstrate that the range of solid forms that may be developed to enhance one or more physicochemical properties of molecular compounds is wider than previously thought. Computational methods remain indispensable in supporting the discovery of new functional crystalline forms of organic compounds.

[1] Reilly, A. M. et al. (2016). Acta Crystallogr., Sect. B: Struct. Sci., Cryst. Eng. Mater. 72, 439-459.

[2] Shunnar, A. F., Dhokale, B., Karothu, D. P., Bowskill, D. H., Sugden, I. J., Hernandez, H. H., Naumov, P. \& Mohamed, S. (2020). Chemistry - A European Journal 26, 4752-4765.

[3] Saeed, Z. M., Dhokale, B., Shunnar, A. F., Awad, W. M., Hernandez, H. H., Naumov, P. \& Mohamed, S. (2021). Cryst. Growth Des. 21, 41514161 .

Keywords: crystal structure prediction; solid-form discovery; in-silico methods; eutectic composites; higher-order cocrystals Acta Cryst. (2021), A77, C592 